\title{
Stability Analysis of the Natural Field Orientation Controlled Induction Machine Drive
}

\author{
G. Mirzaeva ${ }^{\dagger}$ and A. Rojas* \\ School of Electrical Engineering and Computer Science \\ The University of Newcastle, Callaghan, 2308 NSW, Australia \\ Phone: +61 (2) $49215963^{\dagger}$; +61 (2) 49216023* \\ Email: Galina.Mirzaeva@newcastle.edu.au ${ }^{\dagger}$; Alejandro.Rojas@newcastle.edu.au*
}

\section{KEYWORDS}

Vector control, Variable speed drive, Control of drive, Induction motor.

\section{ABSTRACT}

Natural Field Orientation (NFO) Control proposed in the 1980 's is an alternative low cost control method for induction machines. The NFO Control has shown to have some stability issues in regeneration which have been partially addressed in the previous papers. In the present paper a linearised stability analysis is presented for the motor/drive system based on both the non-augmented and the augmented versions of the NFO control. Based on the results of this analysis, the limitations of the augmentation strategy are explored and conditions are identified when it does not ensure stability. Theoretical results are confirmed by simulations and experiments. Directions of further improvement of the NFO stability are outlined.

\section{INTRODUCTION}

Natural Field Orientation (NFO) is a patented control strategy proposed in 1980's ( [1], [2]). It belongs to the Stator Flux Orientation (SFO) control family and is primarily distinguished by that the magnitude of the stator flux vector is not obtained by integration but is assumed to be equal to its reference value. Consider the stator voltage equation in the stator flux oriented reference frame (denoted by the index " $\left.\psi_{s} "\right)$ :

$$
\underline{e}_{s \psi_{s}}=\underline{u}_{s \psi_{s}}-R_{s} \underline{i}_{s \psi_{s}}=\frac{d\left|\psi_{s}\right|}{d t}+j \omega_{m s}\left|\psi_{s}\right|
$$

where $\left|\psi_{s}\right|$ is the magnitude of the stator flux vector $\underline{\psi}_{s} ; R_{s}$ is stator resistance; $\omega_{m s}$ is angular velocity of the stator flux vector $\psi_{s}$. Splitting equation (1) into real and imaginary parts yields:

$$
\begin{aligned}
& e_{s x}=u_{s x}-R_{s} i_{s x}=\frac{d\left|\psi_{s}\right|}{d t}=L_{m} \frac{d\left|i_{m s}\right|}{d t} \\
& e_{s y}=u_{s y}-R_{s} i_{s y}=\omega_{m s}\left|\psi_{s}\right|=\omega_{m s} L_{m}\left|i_{m s}\right|
\end{aligned}
$$

where $x$-axis is aligned with and $y$-axis is in quadrature to the vector $\psi_{s} ; L_{m}$ is magnetising inductance; $\left|i_{m s}\right|$ is the magnitude of the stator magnetising current.
The magnitude of the stator flux vector can be obtained from (2) by integrating the estimated value of $e_{s x}$. The angular velocity of the stator flux vector can then be found from (3). The NFO control technique suggests to exclude the flux integrator and to assume that $\left|\psi_{s}\right|$ equals to its reference value $\left|\psi_{s}^{*}\right|$. This assumption results in elimination of the problems commonly associated with the flux integrator (see, for example, [3]), and in acquisition of some new properties as compared to the traditional SFO control (hence a separate name for the NFO control technique).

The property of "natural" frame orientation was explained in [5]. The true position of the stator flux vector and the associated $(x, y)$ reference frame is not known to the algorithm. It uses the estimated stator flux angular position and associates with it a reference frame that we will call $(d, q)$, or the control frame. If the control frame deviates from the true frame than an inplicit mechanism "naturally" present in the NFO algorithm would act to compensate for such misalignment.

It was also shown in [5] that for the original version of the NFO algorithm the natural self-alignment mechanism is limited to the motoring mode of operation only. Under certain conditions in regeneration, in the case of a small initial misalignment, the control frame would drift further away from the true frame and the stability of the algorithm would be lost. An augmentation to the original algorithm was proposed in [5] and [6] that overcomes this limitation (to a certain extent) and ensures stable operation (in the steady state sense only) in regeneration with limited torque.

Another desirable property of the NFO algorithm is its high tolerance of the parameter errors. The only two machine parameters that it needs to know are the stator resistance $R_{s}$ and the magnetising inductance $L_{m}$. It was shown in [4] that the original and, especially, the augmented NFO versions are very little sensitive to the accurate knowledge of both parameters. The NFO algorithm compensates for the parameter errors by introducing a small angular misalignment between the control and the true reference frames, which is usually very small and does not compromise the stable operation.

The purpose of this paper is to present a more comprehensive stability analysis of the NFO-controlled induction machine including the dynamic effects. Moreover, the analysis will include not only the frame angular error but the close-loop 


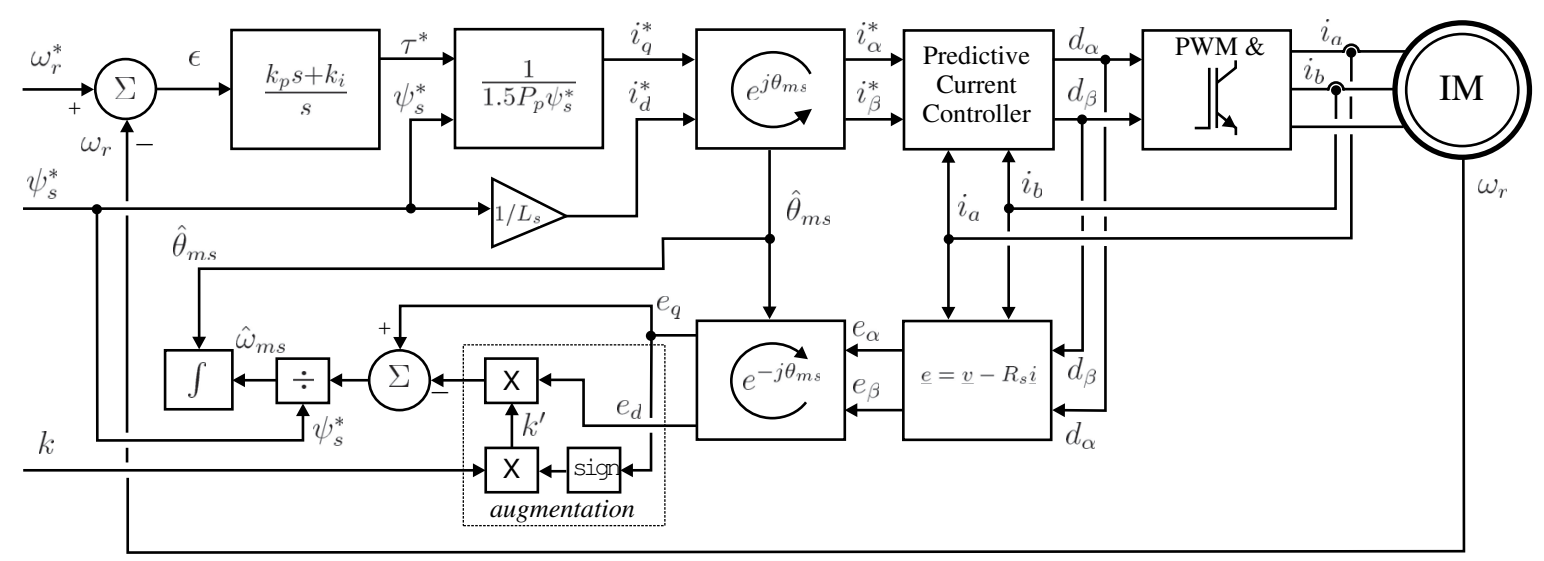

Fig. 1: Block diagram of the NFO algorithm

operation of the whole system from the speed controller to the mechanical load. The primary motivation of this detailed analysis is to explore the limits of the proposed stabilisation strategy in order to:

- conclude whether or not the NFO stability problem, in principle, can be fixed using the proposed augmentation strategy, or other solutions should be sought for;

- make sure that all the dynamic effects possibly affecting stability are included in the analysis.

The remainder of this synopsis is organised as follows. First, a model of the motor / drive system based on NFO control is developed. Small signal linearisation is then performed for the non-augmented (basic) and the augmented versions of the NFO algorithm. Following this, stability analysis based on the root locus method is presented for the linearised model. Simulation and experimental results are included to support the main theoretical conclusions. Finally, conclusions are made and the main contributions of the paper are listed together with an outline of future work.

\section{Model DeVElopMent}

The block diagram of Fig. 1 further assists in understanding of the NFO control algorithm. Indices $\alpha, \beta$ in this figure denote variables in the stationary reference frame. Indices $d, q$, as defined previously, refer to the rotating frame aligned with the estimated position of the stator flux vector (or the control frame). Conversion from one frame to another is performed via the estimated angular position $\hat{\theta}_{m s}$ of the stator flux vector, which is obtained by integrating the angular velocity $\hat{\omega}_{m s}$.

It was shown in [7] that current control of the inverter on both $d$ and $q$ axes is the preferred control option for the NFO implementation. It is only with the current-controlled inverter that the proposed augmentation strategy can be implemented. In Fig. 1 one can see the predictive current control loops around the inverter on both axes.

The optional augmentation branch enclosed in Fig. 1 in a dotted line box is based on the following idea. In the case that the stator flux magnitude is constant or changes slowly then according to (2) the direct axis back emf $e_{s x}$ should be close to zero. If the true and control frames are coincident, the correct stator resistance value is used and the stator voltages and currents are measured accurately, then the estimated direct axis back emf $e_{d}=u_{d}-R_{s} i_{d}$ equals to $e_{s x}$ and is also close to zero.

In fact, the main reasons why $e_{d}$ can notably differ from zero are the frame misalignment and the stator resistance error. Leaving the $R_{s}$ error aside for a moment, one can use the nonzero $e_{d}$ value as the indicator of the frame misalignment to adjust the position of the control frame. This can be done via the following augmentation of the frame angular velocity estimation:

$$
\hat{\omega}_{m s}=\frac{e_{q}-k^{\prime} e_{d}}{\psi_{s}^{*}}
$$

where $k^{\prime}=k \operatorname{sign}\left(e_{q}\right)$, the factor $\operatorname{sign}\left(e_{q}\right)$ takes into account the direction of mechanical rotation and $k>0$ is a tunable gain parameter. The augmentation (4) was first proposed in [5]. With relation to the $R_{s}$ error, it was shown in [4] that the augmentation (4) also improves the NFO tolerance to this commonly problematic error.

Note that if $k=0$ then one has the original (i.e. nonaugmented) NFO algorithm. The further model development will be based on the augmented NFO version with the original NFO algorithm being its special case at $k=0$. For the speed loop analysis it can be assumed that the inner current control loop is very fast and ensures that the reference currents are followed precisely: $i_{\alpha}=i_{\alpha}^{*}$ and $i_{\beta}=i_{\beta}^{*}$. Assume that angular position of the stator flux vector is estimated with an error $\theta_{e}$ relative to its actual position, i.e. that $\hat{\theta}_{m s}=\theta_{m s}+\theta_{e}$. Then it follows that the stator currents in the true reference frame $(x, y)$ relate to those defined in the control frame $(d, q)$ as per:

$$
\begin{aligned}
& i_{s x}=i_{d}^{*} \cos \theta_{e}-i_{q}^{*} \sin \theta_{e} \\
& i_{s y}=i_{d}^{*} \sin \theta_{e}+i_{q}^{*} \cos \theta_{e}
\end{aligned}
$$


Likewise, given that the stator currents and voltages are measured accurately in the stationary frame and the correct $R_{s}$ value is used, the back emfs estimated in the control and the actual back emfs defined in the true frame have the following relation:

$$
\begin{aligned}
& e_{d}=e_{s x} \cos \theta_{e}+e_{s y} \sin \theta_{e} \\
& e_{q}=-e_{s x} \sin \theta_{e}+e_{s y} \cos \theta_{e}
\end{aligned}
$$

Using equation (6), electromagnetic torque can be expressed as:

$$
\tau_{e m}=\frac{3}{2} P_{p} L_{m}\left|i_{m s}\right|\left(i_{d}^{*} \sin \theta_{e}+i_{q}^{*} \cos \theta_{e}\right)
$$

To obtain a more compact torque expression, the following notations have been introduced: $\tau_{0}^{*}$ is the reference torque for the case that $i_{q}^{*}=i_{d}^{*} ; x=\tau_{e m}^{*} / \tau_{0}^{*}=i_{q}^{*} / i_{d}^{*}$ is the normalised reference torque or, equally, the ratio of the current reference values; $\tau_{n}=\tau_{e m} / \tau_{0}^{*}$ is the normalised actual torque; and $y=L_{m}\left|i_{m s}\right| /\left(L_{s} i_{d}^{*}\right)$ is the normalised stator flux magnitude. Adopting these notations one obtains:

$$
\tau_{n}=y\left(\sin \theta_{e}+x \cos \theta_{e}\right)
$$

Using (2) and (3), equations (7) and (8) transform into:

$$
\begin{aligned}
& e_{d}=L_{m} \frac{d\left|i_{m s}\right|}{d t} \cos \theta_{e}+\omega_{m s} L_{m}\left|i_{m s}\right| \sin \theta_{e} \\
& e_{q}=-L_{m} \frac{d\left|i_{m s}\right|}{d t} \sin \theta_{e}+\omega_{m s} L_{m}\left|i_{m s}\right| \cos \theta_{e}
\end{aligned}
$$

To find the expression for $L_{m}\left|i_{m s}\right|$ (and consequently, for $y$ ) we will use the rotor voltage equations in the stator flux frame, which real and imaginary parts can be manipulated into (see, for example, [3]):

$$
\begin{gathered}
L_{m} \frac{d\left|i_{m s}\right|}{d t}+\frac{L_{m}}{T_{r}}\left|i_{m s}\right|=\sigma L_{s} \frac{d i_{s x}}{d t}+\frac{L_{s}}{T_{r}} i_{s x}-\sigma L_{s} \omega_{s l} i_{s y} \\
\omega_{s l}\left(L_{m}\left|i_{m s}\right|-\sigma L_{s} i_{s x}\right)=\sigma L_{s} \frac{d i_{s y}}{d t}+\frac{L_{s}}{T_{r}} i_{s y}
\end{gathered}
$$

where $L_{m}$ and $L_{s}$ are the magnetising and the stator inductances respectively; $T_{r}$ is the rotor time constant; $\omega_{s l}$ is the slip frequency of the rotor with relation to the stator flux vector; and $\sigma$ is the machine leakage constant.

Substituting expressions for $i_{s x}, i_{s y}$ and their derivatives from (5) and (6), and using the previously introduced normalised variables $x$ and $y$, equations (13) and (14) can be manipulated into:

$$
\begin{aligned}
\frac{d y}{d t}+\frac{1}{T_{r}} y & =\frac{1}{T_{r}}\left(\cos \theta_{e}-x \sin \theta_{e}\right) \\
& -\sigma \frac{d \theta_{e}}{d t}\left(\sin \theta_{e}+x \cos \theta_{e}\right)-\sigma \frac{d x}{d t} \sin \theta_{e} \\
& -\sigma \omega_{s l}\left(\sin \theta_{e}+x \cos \theta_{e}\right)
\end{aligned}
$$

$\omega_{s l}=$

$\frac{\frac{1}{T_{r}}\left(\sin \theta_{e}+x \cos \theta_{e}\right)+\sigma \frac{d \theta_{e}}{d t}\left(\cos \theta_{e}-x \sin \theta_{e}\right)+\sigma \frac{d x}{d t} \cos \theta_{e}}{y-\sigma\left(\cos \theta_{e}-x \sin \theta_{e}\right)}$
Now, the angular misalignment between the true and the control reference frames can be expressed as follows:

$$
\frac{d \theta_{e}}{d t}=\omega_{e}=\hat{\omega}_{m s}-\omega_{m s}=\hat{\omega}_{m s}-P_{p} \omega_{r}-\omega_{s l}
$$

where $\omega_{e}$ is error in the frame angular velocity estimation; $\omega_{r}$ is the rotor angular velocity; and $P_{p}$ is the number of pole pairs. Using the augmented estimation (4) of the frame angular velocity $\hat{\omega}_{m s}$, together with expressions (11) and (12) for $e_{d}$ and $e_{q}$, one obtains:

$$
\hat{\omega}_{m s}=\omega_{m s} y\left(\cos \theta_{e}-k^{\prime} \sin \theta_{e}\right)-\frac{d y}{d t}\left(\sin \theta_{e}+k^{\prime} \cos \theta_{e}\right)
$$

substituting which into the angular misalignment equation (17) yields:

$$
\begin{aligned}
\frac{d \theta_{e}}{d t}=\omega_{e} & =\left(P_{p} \omega_{r}+\omega_{s l}\right)\left[y\left(\cos \theta_{e}-k^{\prime} \sin \theta_{e}\right)-1\right] \\
& -\frac{d y}{d t}\left(\sin \theta_{e}+k^{\prime} \cos \theta_{e}\right)
\end{aligned}
$$

Finally, to complete the system description, the equation of mechanical rotation is added:

$$
\frac{d \omega_{r}}{d t}=\frac{\tau_{0}^{*}}{J} \tau_{n}
$$

where $J$ is the total mechanical intertia of the machine and the load. Other load torque components not related to the intertia are treated as a disturbance and are not included in the equation (20). This simplified load model can be replaced, if needed, by any other practical load model.

Equations (10), (15), (16), (19) and (20) fully characterise the dynamic behaviour of the NFO controlled induction machine. A separate equation (16) for $\omega_{s l}$ is kept for the sake of shorter expressions. It will be substituted into (15) and (19) when practical, and thus the variable $\omega_{s l}$ will be eliminated. Apparently, the system is non-linear. For an easier understanding of its structure, the system equations can be written in the following way:

$$
\begin{gathered}
\frac{J}{\tau_{0}^{*}} \frac{d \omega_{r}}{d t}=\tau_{n}=f_{1}\left(x, y, \theta_{e}\right) \\
\frac{d y}{d t}=f_{2}\left(\frac{d \theta_{e}}{d t}, \frac{d x}{d t}, \theta_{e}, x, y\right) \\
\frac{d \theta_{e}}{d t}=\omega_{e}=f_{3}\left(\frac{d y}{d t}, \frac{d x}{d t}, y, x, \omega_{r}, \theta_{e}\right)
\end{gathered}
$$

where functions $f_{1}, f_{2}$ and $f_{3}$ are defined, respectively, in (10), (15) and (19). A block diagram of the control system under the NFO control is shown in Fig. 2. One can see from Fig. 2 that the structure of the control system is straightforward, except for a complicated non-linear feedback present in the model of the NFO algorithm.

It would be tempting to describe the system in terms of a general state variable model, with $\omega_{r}^{*}$ as its input; $\omega_{r}$ as its output; and $x, y, \omega_{r}$ and $\theta_{e}$ as its state variables. However, we will refrain from using these terms as the classical definition of non-linear state equations (see, for example, [8]) requires 


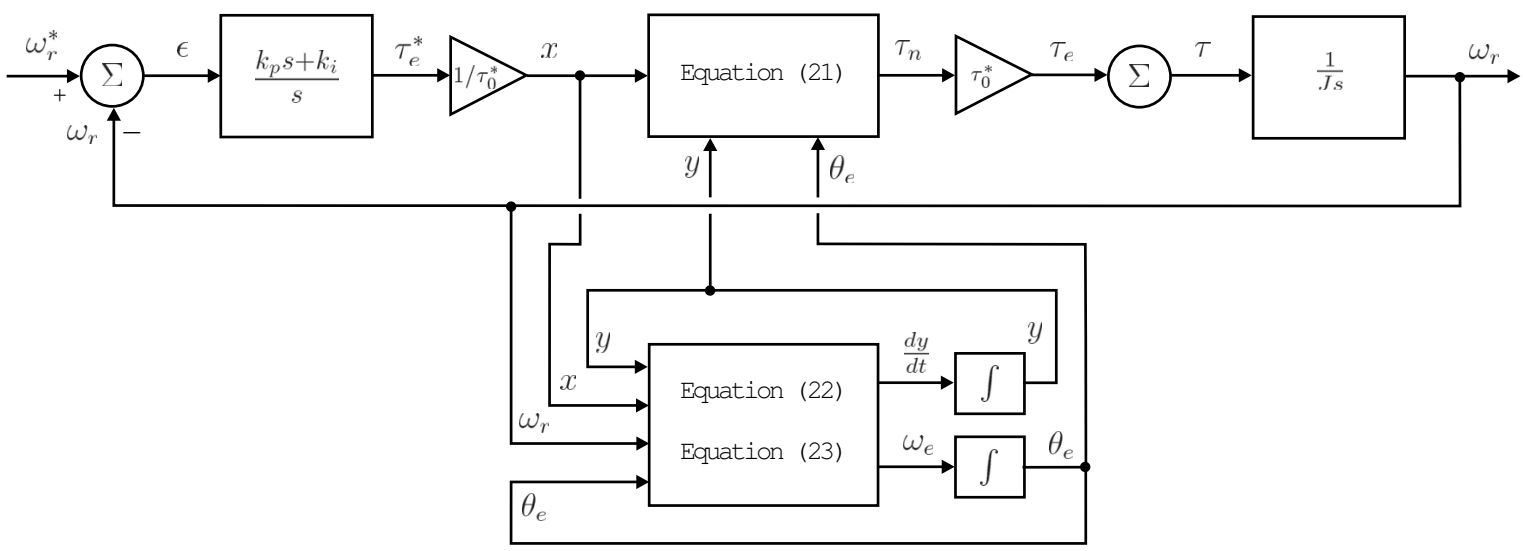

Fig. 2: Block diagram of the control system under the NFO control

that no derivatives are present in their right-hand side. We would rather say that the variables $x, y, \omega_{r}$ and $\theta_{e}$ are like the state variables and equations (21)-(23) are similar to the state equations of the system.

\section{SMALl Signal LinEARISATION AND StABILITY ANALYSIS}

Based on the model developed in the previous section, we will now perform a small signal linearisation of the system equations and develop linearised transfer functions of the system. The following transfer functions will be of particular interest:

- Transfer function from the commanded torque $\tau_{e m}^{*}$ to the actual torque $\tau_{e m}$, or, equally, between their normalised values $x$ and $\tau_{n}$. This transfer function will determine the behaviour of the NFO algorithm (plant) itself, without the PI controller. The model of the plant will include its own internal feedback from the output variable $\omega_{r}$.

- Transfer function from the commanded angular velocity $\omega_{r}^{*}$ to the output angular velocity $\omega_{r}$. This transfer function will include the contribution of the PI controller and will determine the close loop behaviour of the whole system under the NFO control.

- Transfer function from the angular velocity error $\epsilon=$ $\omega_{r}^{*}-\omega_{r}$ to the output angular velocity $\omega_{r}$. This open loop transfer function is practical to use with the Root Locus method of stability analysis.

Applying small signal linearisation, in the neighborhood of a chosen operation point, to non-linear equations (21)-(23) results in the expressions of the following form:

$$
\begin{gathered}
\delta \tau_{n}=\alpha_{1} \delta x+\alpha_{2} \delta y+\alpha_{3} \delta \theta_{e} \\
\delta \dot{y}=\alpha_{4} \delta \dot{\theta}_{e}+\alpha_{5} \delta \dot{x}+\alpha_{6} \delta \theta_{e}+\alpha_{7} \delta x+\alpha_{8} \delta y \\
\delta \dot{\theta}_{e}=\alpha_{9} \delta \dot{y}+\alpha_{10} \delta \dot{x}+\alpha_{11} \delta y+\alpha_{12} \delta x+\alpha_{13} \delta \omega_{r}+\alpha_{14} \delta \theta_{e}
\end{gathered}
$$

where $\alpha_{1}-\alpha_{14}$ are linearisation coefficients dependent on the operating point. By applying Laplace transform to expressions (24)-(26) and manipulating to exclude $\delta y(s)$ and $\delta \theta_{e}(s)$, one obtains the following transfer function for $\delta \tau_{n}(s)$ with relation to $\delta x(s)$ :

$$
\frac{\delta \tau_{n}(s)}{\delta x(s)}=\frac{J s\left(a_{2} s^{2}+a_{1} s+a_{0}\right)}{\tau_{0}^{*}\left(b_{3} s^{3}+b_{2} s^{2}+b_{1} s+b_{0}\right)}
$$

where $a_{0}-a_{2}$ and $b_{0}-b_{3}$ are bulky expressions in terms of $\alpha_{1}$ $\alpha_{14}$ omitted here for the sake of space. Now, based on the control structure of Fig. 2 the open loop transfer function of the system can be found as:

$$
\frac{\delta \omega_{r}(s)}{\delta \epsilon(s)}=\frac{k_{p}}{\tau_{0}^{*}} \frac{\left(a_{2} s^{2}+a_{1} s+a_{0}\right)\left(s+k_{i} / k_{p}\right)}{s\left(b_{3} s^{3}+b_{2} s^{2}+b_{1} s+b_{0}\right)}
$$

where $k_{p}$ and $k_{i}$ are parameters of the PI controller. Finally, the close loop transfer function of the system is given by:

$$
\begin{aligned}
& \frac{\delta \omega_{r}(s)}{\delta \omega_{r}^{*}(s)}=\frac{\frac{k_{p}}{\tau_{0}^{*}}\left(a_{2} s^{2}+a_{1} s+a_{0}\right)\left(s+k_{i} / k_{p}\right)}{\frac{k_{p}}{\tau_{0}^{*}}\left(a_{2} s^{2}+a_{1} s+a_{0}\right)\left(s+k_{i} / k_{p}\right)+} \\
& \overline{+s\left(b_{3} s^{3}+b_{2} s^{2}+b_{1} s+b_{0}\right)}
\end{aligned}
$$

These transfer functions will be now examined for stability using classical tools such as Evans's root locus method and analysis of polynomial coefficients of the transfer function.

\section{A. Non-augmented NFO}

Firstly, we study the stability of the non-augmented NFO where the augmentation gain in (4) $k^{\prime}=0$. The expressions for coefficients $a_{0}-a_{2}$ and $b_{0}-b_{3}$ in equation (28) are very bulky and are not practical to be presented in this paper. It is however possible to determine, with a reasonable degree of accuracy, what parameters of the system dominate each of these coefficients and under what conditions they may turn negative. Such approximate expressions for the case when 
$k^{\prime}=0$ are given below:

$$
\begin{aligned}
a_{2} & \approx y ; \quad a_{1} \approx y\left(\frac{1}{T_{r}}+\sigma x P_{p} \omega_{r}\right) \\
a_{0} & \approx \frac{y}{T_{r}}\left(P_{p} \omega_{r} x-\frac{x^{2}}{y^{2} T_{r}}-\frac{x^{2}}{y T_{r}}-\frac{1}{T_{r}}\right) \\
b_{3} & \approx \frac{J}{\tau_{0}^{*}} ; \quad b_{2} \approx \frac{J}{\tau_{0}^{*}}\left(\frac{1}{T_{r}}+\sigma x P_{p} \omega_{r}\right) \\
b_{1} & \approx \frac{J}{\tau_{0}^{*} T_{r}}\left(P_{p} \omega_{r} x-\frac{x^{2}}{y^{2} T_{r}}\right) \\
b_{0} & \approx \frac{P_{p}}{T_{r}}(y-1)\left(x^{2}-y\right)
\end{aligned}
$$

The following observations can be made from examining expressions (30)-(34):

1) Coefficients $a_{2}$ and $b_{3}$ are always positive;

2) Coefficients $a_{1}$ and $b_{2}$ are typically positive but can be negative when regenerating at high speed and torque;

3) Coefficients $a_{0}$ and $b_{1}$ can become negative when motoring at slow speed ( $x$ and $\omega_{r}$ are of the same sign, $\left|\omega_{r}\right|$ is small), and will be negative in regeneration ( $x$ and $\omega_{r}$ are of different signs);

4) Coefficient $b_{0}$, in both motoring and regeneration, can be positive or negative depending on flux and torque magnitudes.

Based on the above observations, the following open loop performance is expected from the non-augmented NFO algorithm. In motoring the algorithm can be stable given that torque is appropriately limited and that the relation between $y$ and $x$ ensures that $b_{0}>0$. The latter condition is not guaranteed but may be possible due to the natural frame alignment property, i.e. when the algorithm naturally finds a misaligned frame position corresponding to a stable operation point. In regeneration the algorithm is expected to be unstable under most conditions. As it will be shown in the next section, these expectations agree to simulation and experimental results.

Now, using the root locus method, we will explore how the gain of the PI controller affects the system stability. To vary the controller gain we will change $k_{p}$ from zero to infinity while keeping the ratio $k_{i} / k_{p}$ constant. Fig. 3 a shows the root locus of the transfer function (28) in motoring mode. The operating point here is defined by $\omega_{r}=100 \mathrm{rad} / \mathrm{sec} ; x=0.8 ; y=0.92$; and $\theta_{e}=-0.05 \mathrm{rad}$. On can see that the system is stable under all non-zero gains of the PI-controller.

Another motoring example is shown in Fig. 3b. The operating point differs from the previous case in that $\omega_{r}=$ $1 \mathrm{rad} / \mathrm{sec}$. At low gain values of the controller there exist a pair of complex poles in the right half plane (RHP) and a pole at the origin. As the gain increases, the complex poles are pulled into the left half plane (LHP) but the pole at the origin moves along the real axis into the RHP. This indicates that when motoring at very slow speed the system is unstable.

Regeneration mode of the non-augmented NFO is illustrated by Fig. $3 \mathrm{c}$ for $\omega_{r}=100 \mathrm{rad} / \mathrm{sec} ; x=-0.8 ; y=0.92$; $\theta_{e}=0.05 \mathrm{rad}$. For any gain of the PI-controller there exists a root in the RHP, which indicates a severe stability problem.

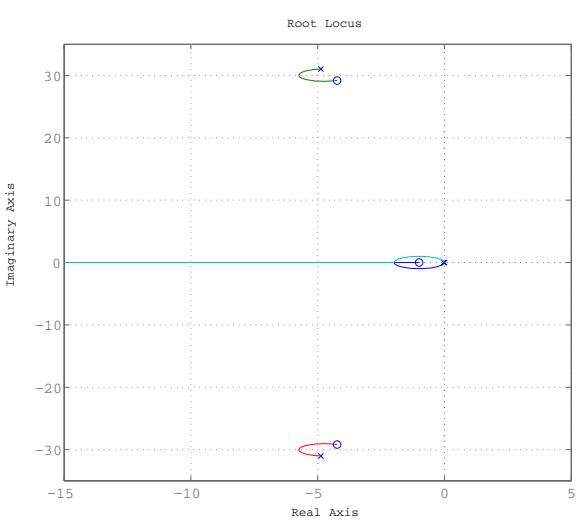

(a) Motoring, stable operation

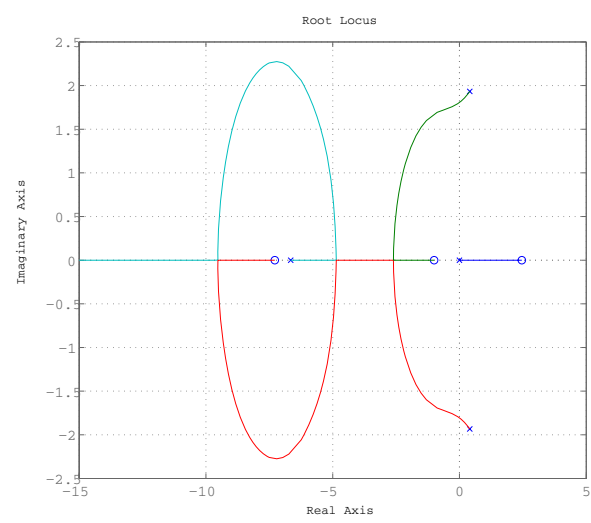

(b) Motoring, unstable operation at low speed

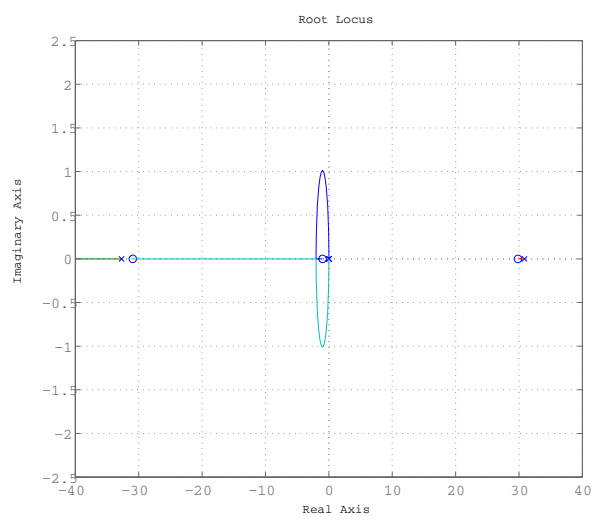

(c) Regeneration, unstable operation

Fig. 3: Root locus of the NFO controlled system, nonaugmented algorithm 


\section{B. Augmented NFO}

The same analysis will be now applied to the augmented version of the NFO algorithm. The approximate expressions for the polynomial coefficients for the case when $k^{\prime} \neq 0$ is now given by:

$$
\begin{aligned}
a_{2} & \approx y ; \quad a_{1} \approx y\left(\frac{1}{T_{r}}+\sigma x P_{p} \omega_{r}+k^{\prime} y P_{p} \omega_{r}\right) \\
a_{0} & \approx \frac{y}{T_{r}}\left(P_{p} \omega_{r} x-\frac{x^{2}}{y^{2} T_{r}}-\frac{x^{2}}{y T_{r}}-\frac{1}{T_{r}}+k^{\prime} y P_{p} \omega_{r}+k^{\prime} \frac{x}{T_{r}}\right) \\
b_{3} & \approx \frac{J}{\tau_{0}^{*}} ; \quad b_{2} \approx \frac{J}{\tau_{0}^{*}}\left(\frac{1}{T_{r}}+\sigma x P_{p} \omega_{r}+k^{\prime} y P_{p} \omega_{r}\right) \\
b_{1} & \approx \frac{J}{\tau_{0}^{*} T_{r}}\left(P_{p} \omega_{r} x-\frac{x^{2}}{y^{2} T_{r}}+k^{\prime} y P_{p} \omega_{r}+k^{\prime} \frac{x}{T_{r}}\right) \\
b_{0} & \approx \frac{P_{p}}{T_{r}}(y-1)\left(x^{2}-y\right)
\end{aligned}
$$

The following can be observed from examining expressions (35)-(39):

1) Coefficients $a_{2}$ and $b_{3}$ are always positive;

2) Due to introduction of the term $k^{\prime} y P_{p} \omega_{r} y$, which is always positive, coefficients $a_{1}$ and $b_{2}$ are typically positive;

3) Due to introduction of the same positive term, coefficients $a_{0}$ and $b_{1}$ can be kept positive under any conditions by using $k$ of an appropriate magnitude;

4) The proposed augmentation had practically (within the accuracy of the approximation) no influence on the coefficient $b_{0}$ which, as in the non-augmented case, can be positive or negative depending on the flux and torque magnitudes.

From the above observations it follows that the augmentation has a positive effect on the system stability. When the speed loop is open, given that $b_{0}>0$, the NFO algorithm is expected to be stable when motoring and when regenerating with limited torque. If the gain $k$ is allowed to change depending on the speed and torque then the region of stable regeneration can be further extended. However, the proposed augmentation has added practically no control over the sign of $b_{0}$. Therefore, with the speed loop open, there is a possibility that the NFO algorithm can be unstable regardless of the $k$ value.

This can be illustrated with the help of the following example. The operating point of the system is chosen as: $\omega_{r}=100 \mathrm{rad} / \mathrm{sec} ; x=-0.8 ; y=0.92 ; \theta_{e}=-0.05 \mathrm{rad}$. Under these conditions, the non-augmented NFO algorithm is unstable as all the polynomial coefficients, except for $a_{2}$ and $b_{3}$, are negative. Augmentation with $k=1$ leads to $a_{1}$ and $b_{2}$ turning positive. Increasing the gain to $k=1.5$ makes $a_{0}$ and $b_{1}$ positive, but even then $b_{0}$ remains negative.

If the speed loop is closed then, with the appropriate gain of the PI controller, the system becomes stable. This is illustrated by the root locus of Fig. $4 \mathrm{~b}$ plotted for the above example with $k=1.5$. Fig. $4 \mathrm{c}$ shows a magnified view of the same plot near

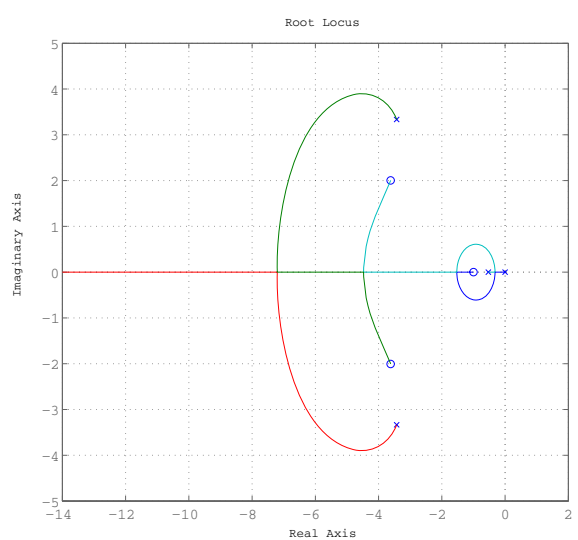

(a) Motoring, stable operation at low speed

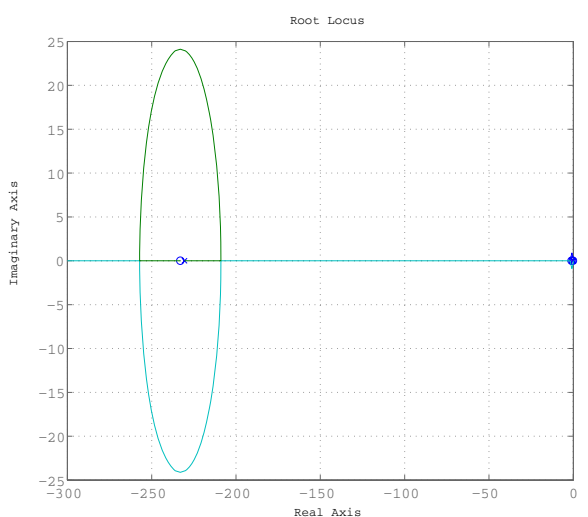

(b) Regeneration

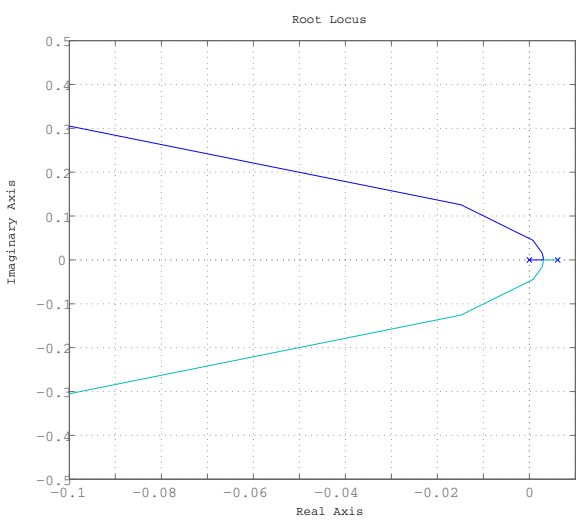

(c) Regeneration, magnified view

Fig. 4: Root locus of the NFO controlled system, augmented algorithm with $k=1$ 
the origin. It can be seen how, as the $k_{p}$ grows, the negative pole and the pole in the origin move towards each other along the real axis in the RHP, then break into a pair of complex poles and are pulled into the LHP. Fig. 4a illustrates stable motoring at very slow speed under the same conditions as those of Fig. $3 \mathrm{~b}$ except that $k=1$.

\section{SIMULATION AND EXPERIMENTAL RESULTS}

To illustrate the stability problem, performance of the NFO controlled induction machine was simulated under the following conditions: after allowing $0.5 \mathrm{sec}$ for fluxing, the reference speed stepped up from zero to $100 \mathrm{rad} / \mathrm{sec}$ and then, after $1 \mathrm{sec}$ stepped down back to zero. The accurate values of the stator resistance and the magnetising inductance were used by the algorithm. Fig. 5a shows simulation results for the nonaugmented NFO control and includes plots for the mechanical speed (desired vs actual), electromagnetic torque (desired vs actual) and the angular misalignment of the control frame.

It can be seen from Fig. 5a that in motoring the algorithm showed acceptable performance but immediately after entering regeneration mode NFO lost control and the system became unstable. In this simulation example, the induction motor was driving a highly inertial load, hence for a relatively long time, from $t=0.5 \mathrm{sec}$ to $t=1.25 \mathrm{sec}$, the output of the PI controller was limited at its maximum positive value, i.e. the NFO algorithm was operating with the speed loop open. The same was true for the regeneration part of the plot, from $t=1.5 \mathrm{sec}$ to $t=2.4 \mathrm{sec}$.

The corresponding experimental results showing the same variables appear in Fig. 6a. In general, the results are in a very good agreement with those of the simulation, except for a high initial frame misalignment observed in the experiment. This can be due to the fact that in the experiment we did not know the true frame position, and used as such the position of the stator flux vector estimated by the FOC (working in the background of the NFO).

Simulation results for the augmented algorithm with $k=1$ are shown in Fig. 5b. As compared to the non-augmented case of Fig. 5a, with the augmentation turned on, the algorithm performance in motoring was much better, transition from motoring to regeneration was well controlled. Yet, at some point in regeneration the angular error started growing and stability was lost. The output of the PI controller at this point was limited at its maximum negative value, and the system was operating with the open speed loop.

Experimental results for the augmented algorithm with $k=1$ are shown in Fig. $6 \mathrm{~b}$. The system remained stable in regeneration but only because the negative torque was limited at $-5 \mathrm{Nm}$ rather than $-20 \mathrm{Nm}$ as it was in the corresponding simulation. When a higher negative torque was allowed, the NFO algorithm was losing stability in regeneration in a manner similar to Fig. 5b. High initial frame misalignment seen in the bottom of Fig. $6 \mathrm{~b}$ can be also attributed to the incorrect knowledge of the true frame position at start.

Finally, an attempt was made to further improve the NFO stability by using an augmentation of proportional - integral

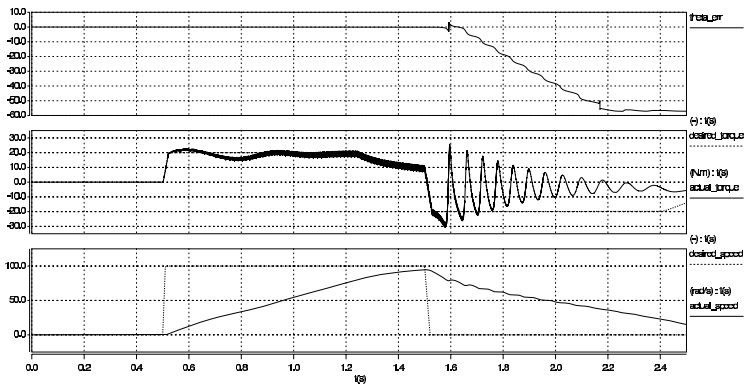

(a) Non-augmented NFO

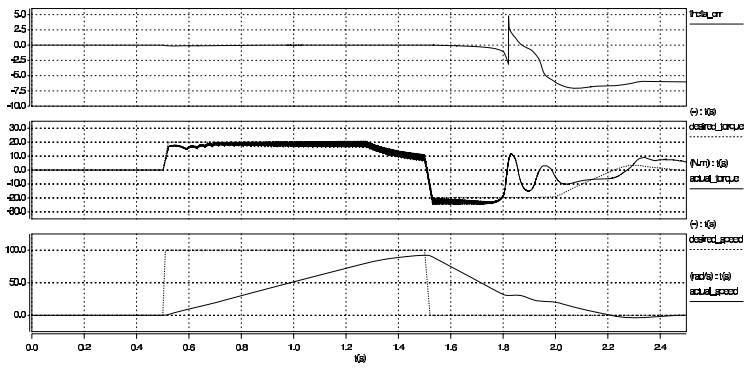

(b) Augmented NFO, $k=1$

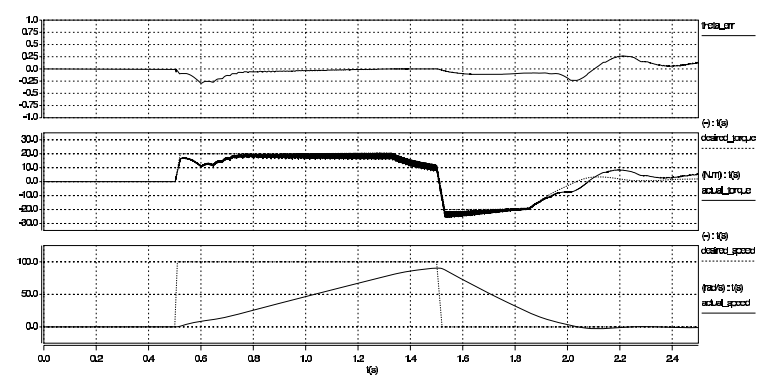

(c) Augmented NFO, $k=1, m=10$

Fig. 5: Simulation plots for different strategies of the NFO control

structure, i.e. estimating the frame angular velocity according to:

$$
\hat{\omega}_{m s}=\frac{e_{q}-k^{\prime} e_{d}-m^{\prime} \int e_{d} d t}{\psi_{s}^{*}}
$$

where $m^{\prime}=m \operatorname{sign}\left(e_{q}\right) ; m$ is the independently controlled integral gain; and the other parameters are as in equation (4). By the time the paper was submitted, the stability analysis of thus augmented system was not completed, and therefore, only simulation and experimental results are included to illustrate this case, as shown in Fig. 5c and Fig. 6c respectively. One can see that with the proportional- integral augmentation stability of the system has improved, the motor starts and stops in a controlled manner, yet some problems are evident around zero speed. 

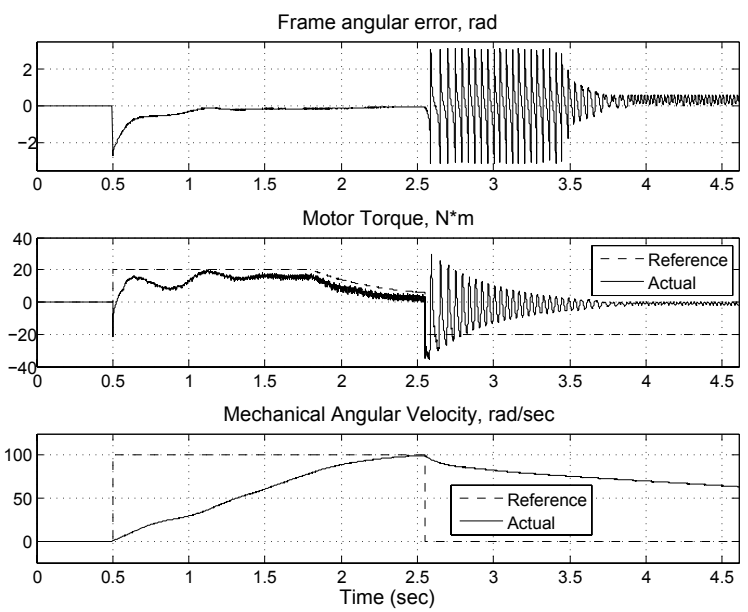

(a) Non-augmented NFO
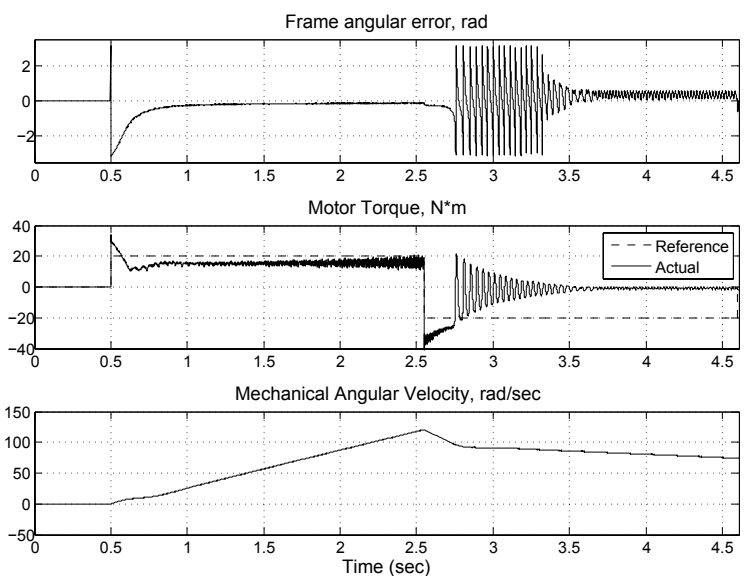

(b) Augmented NFO, $k=1$
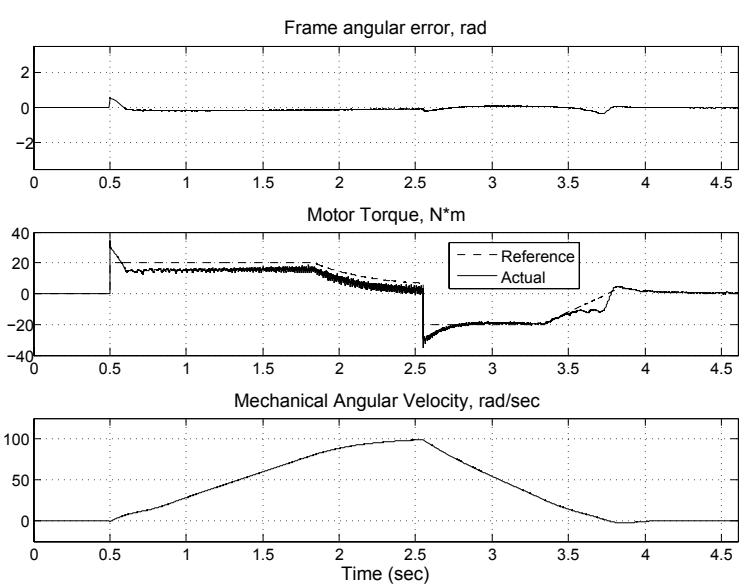

(c) Augmented NFO, $k=1, m=10$

Fig. 6: Experimental plots for different strategies of the NFO control

\section{CONCLUSIONS AND CONTRIBUTIONS}

The main conclusion of the analysis presented in this paper is that the augmentation to the NFO algorithm proposed in [5], may not guarantee the NFO stability under all conditions. This limitation comes from the fact that the algorithm offers no control over one of the essential parameters - the coefficient $b_{0}$ in the denominator of the transfer function. Particular conditions when the stability can be lost include regeneration with the open speed loop, which is likely to occur when a negative step change in speed is commanded, and the output of the PI controller is limited at its maximum negative value.

The following are the main contributions of the paper:

- The full dynamic equations describing the original and the augmented NFO algorithm have been developed.

- Linearised dynamic stability analysis of the full motor/drive system based on the NFO control algorithm has been presented. These results do not contradict but add to the previously presented in [5] static stability analysis.

- Stability problems have been identified and related to the parameters of the system.

- The limits of the augmentation strategy based on the auxiliary $e_{d}$ feedback have been explored. The most critical parameter that limits the stabilisation effect of the strategy has been identified.

- Simulation and experimental results for the nonaugmented and the augmented versions of the NFO algorithm have been presented. These results agree with the conclusions of the stability analysis.

- Preliminary simulation and experimental results for the proportional - integral version of the NFO augmentation have been included. These show improved performance in both motoring and regenration.

In future work the NFO-controlled system with the proportional - integral augmentation will be analysed in detail. It will be also attempted to find alternative stabilisation strategies that would provide stability of the NFO algorithm under an extended range of conditions.

\section{REFERENCES}

[1] R. Jönsson, "Method and apparatus for controlling an AC motor," United States Patent 4,458,193, July 1984.

[2] R. Jönsson, "Method and apparatus for controlling an ac induction motor by indirect measurement of the air-gap voltage," United States Patent 5,294,876, March 1994

[3] P. Vas, Sensorless Vector and Direct Torque Control. Oxford University Press, 2003.

[4] G. Mirzaeva and R. E. Betz, "Parameter sensitivity issues in natural field orientation," in Industry Applications Conference, 2007. 42nd IAS Annual Meeting. Conference Record of the 2007 IEEE, New Orleans, LA, Sep. 23-27, 2007.

[5] R. E. Betz and G. Mirzaeva, "Frame alignment stability issues in natura field orientation," in Industry Applications Conference, 2006. 41st IAS Annual Meeting. Conference Record of the 2006 IEEE, vol. 5, Tampa, FL, Oct. 8-12, 2006, pp. 2411-2418.

[6] G. Mirzaeva and R. E. Betz, "An improved natural field orientation control of a current fed induction machine," in CDROM Proceedings of IEEEIEMDC 2007, Antalya, Turkey, May 2007.

[7] —- "Natural field orientation concept: a tutorial," in CDROM Proceedings of IEEE-IEMDC 2007, Antalya, Turkey, May 2007.

[8] G. C. Goodwin, S. F. Graebe, and M. E. Salgado, Control System Design. Prentice-Hall, 2001. 